\title{
On the optimum spacing of stereoscopic imaging atmospheric Cherenkov telescopes
}

W. Hofmann*a, G. Hermann ${ }^{\mathrm{a}}$, A. Konopelko ${ }^{\mathrm{a}}$, H. Krawczynski ${ }^{\text {a }}$, C. Köhler ${ }^{\text {a }}$, G. Pühlhofer ${ }^{a}$, F.A. Aharonian ${ }^{\text {a }}$, A.G. Akhperjanian ${ }^{\text {b }}$, M. Aye ${ }^{\mathrm{a}}$, J.A. Barrio ${ }^{\mathrm{c}, \mathrm{d}}$, K. Bernlöhr ${ }^{\mathrm{a}, \mathrm{h}}$, J.J.G. Beteta ${ }^{\mathrm{d}}$, H. Bojahr ${ }^{\mathrm{f}}$, J.L. Contreras ${ }^{\mathrm{d}}$, J. Cortina ${ }^{\mathrm{d}}$, A. Daum ${ }^{a}$, T. Deckers ${ }^{\mathrm{e}}$, J. Fernandez ${ }^{\mathrm{c}, \mathrm{d}}$, V. Fonseca ${ }^{\mathrm{d}}$, J.C. Gonzalez ${ }^{\mathrm{d}}$, G. Heinzelmann ${ }^{\mathrm{g}}$, M. Hemberger ${ }^{\mathrm{a}}$,

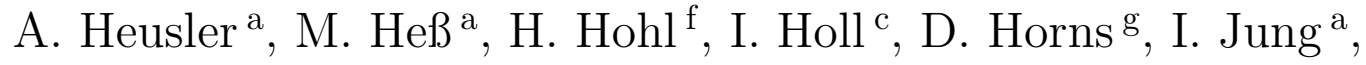
R. Kankanyan ${ }^{\mathrm{a}, \mathrm{b}}$, M. Kestel $^{\mathrm{c}}$, J. Kettler ${ }^{\mathrm{a}}$, A. Kohnle ${ }^{\mathrm{a}}$, H. Kornmayer ${ }^{\mathrm{c}}$, D. Kranich ${ }^{\mathrm{c}}$, H. Krawczynski ${ }^{\mathrm{a}}$, H. Lampeitl ${ }^{\mathrm{a}}$, A. Lindner ${ }^{g}$, E. Lorenz ${ }^{c}$, N. Magnussen ${ }^{\mathrm{f}}$, O. Mang ${ }^{\mathrm{e}}$, H. Meyer ${ }^{\mathrm{f}}$, R. Mirzoyan ${ }^{\mathrm{c}, \mathrm{d}, \mathrm{b}}$, A. Moralejo $^{\mathrm{d}}$, L. Padilla ${ }^{\mathrm{d}}$, M. Panter ${ }^{a}$, R. Plaga ${ }^{\mathrm{c}}$, J. Prahl ${ }^{\mathrm{g}}$, C. Prosch ${ }^{\mathrm{c}}$, G. Rauterberg ${ }^{\mathrm{e}}$, W. Rhode ${ }^{f}$, A. Röhring ${ }^{g}$, M. Samorski ${ }^{e}$, J.A. Sanchez ${ }^{d}$, M. Schilling e, D. Schmele ${ }^{\mathrm{g}}$, F. Schröder ${ }^{\mathrm{f}}$, W. Stamm ${ }^{\mathrm{e}}$, H.J. Völk ${ }^{a}$, B. Wiebel-Sooth ${ }^{\mathrm{f}}$, C.A. Wiedner ${ }^{\mathrm{a}}$, M. Willmer ${ }^{\mathrm{e}}$

\section{HEGRA Collaboration}

${ }^{a}$ Max-Planck-Institut für Kernphysik, P.O. Box 103980, D-69029 Heidelberg, Germany

${ }^{\mathrm{b}}$ Yerevan Physics Institute, Yerevan, Armenia

${ }^{\mathrm{c}}$ Max-Planck-Institut für Physik, Föhringer Ring 6, D-80805 München, Germany

${ }^{\mathrm{d}}$ Facultad de Ciencias Fisicas, Universidad Complutense, E-28040 Madrid, Spain

e Universität Kiel, Inst. für Experim. u Angew. Physik, Olshausenstr.40, D-24118 Kiel, Germany

${ }^{\mathrm{f}}$ BUGH Wuppertal, Fachbereich Physik, Gaußstr.20, D-42119 Wuppertal, Germany

g Universität Hamburg, II. Inst. für Experimentalphysik, Luruper Chaussee 149, D-22761 Hamburg, Germany

${ }^{\mathrm{h}}$ Now at Forschungszentrum Karlsruhe, P.O. Box 3640, 76021 Karlsruhe

*Corresponding author (Werner.Hofmann@mpi-hd.mpg.de Fax +496221 516 603, Phone +496221516 330) 


\begin{abstract}
For stereoscopic systems of imaging atmospheric Cherenkov telescopes (IACTs), a key parameter to optimize the sensitivity for VHE $\gamma$-ray point sources is the intertelescope spacing. Using pairs of telescopes of the HEGRA IACT system, the sensitivity of two-telescope stereo IACT systems is studied as a function of the telescope spacing, ranging from $70 \mathrm{~m}$ to $140 \mathrm{~m}$. Data taken during the 1997 outburst of Mrk 501 are used to evaluate both the detection rates before cuts, and the sensitivity for weak signals after cuts to optimize the significance of signals. Detection rates decrease by about $1 / 3$ between the minimum and maximum spacing. The significance of signals is essentially independent of distance, in the range investigated.
\end{abstract}

\title{
1 Introduction
}

IACT stereoscopy - the simultaneous observation of air showers with multiple imaging atmospheric Cherenkov telescopes (IACTs) under different viewing angles - has become the technique of choice for most of the next-generation instruments for earth-bound gamma-ray astronomy in the VHE energy range, such as VERITAS[1] or HESS[2]. The stereoscopic observation of air showers allows improved determination of the direction of the primary and of its energy, as well as a better suppression of backgrounds, compared to single IACTs. A crucial question in the layout of stereoscopic systems of IACTs is the spacing of the identical telescopes. Obviously, the spacing of telescopes should be such that at least two telescopes fit into the Cherenkov light pool with its typical diameter of around $250 \mathrm{~m}$. For higher energy showers the light pool can increase considerably, but at large distances $(>130 \mathrm{~m})$ one observes primarily light from shower particles scattered at larger angles. The coincidence rate between two telescopes will decrease with increasing spacing; on the other hand, the angle between the views and hence the quality of the stereoscopic reconstruction of the shower geometry will improve. The opinions within the IACT community concerning the optimum geometry of IACT arrays differ; the HESS project, for example, initially aimed for a spacing of $100 \mathrm{~m}$ between adjacent telescopes; more recently, larger distances around $120 \mathrm{~m}$ or more are favored [3,4]. For the otherwise similar VERITAS array, intertelescope distances of initially $50 \mathrm{~m}$, and later $80 \mathrm{~m}$ to $85 \mathrm{~m}$ were foreseen. Among existing systems, the HEGRA 5-telescope IACT system has a characteristic spacing of $70 \mathrm{~m}$ between its central telescope and the corner telescopes. The WHIPPLE-GRANITE two-telescope system [5] had a spacing of $140 \mathrm{~m}$. The Telescope Array [6] uses a spacing of $120 \mathrm{~m}$ between the first three telescopes, and later $70 \mathrm{~m}$ for the full array.

Optimization of IACT system geometry is heavily based on Monte-Carlo simulations. Over the last years, the quality of these simulations has improved significantly, both concerning the reliability of the shower simulation [7] and concerning the details of the simulation of the telescopes and their readout (see, e.g, [8]). Simulations have been tested extensively, and key characteristics, such as the radial distribution of Cherenkov light in the light pool, have been verified experimentally [9]. Nevertheless, a direct experimental verification of the dependence 
of the performance of IACT systems on the intertelescope distance would be highly desirable.

The HEGRA IACT system at the Observatorio del Roque de los Muchachos on La Palma consists of five telescopes, four of them arranged roughly in the form of a square with $100 \mathrm{~m}$ side length, with the fifth telescope in the center. Selecting pairs of telescopes, the performance of two-telescope stereo systems can be studied for distances between $70 \mathrm{~m}$ (from the central telescope to the corner telescopes) and $140 \mathrm{~m}$ (across the diagonal of the square), covering essentially the entire range of interest. This paper reports the results of such a study, based on the large sample of gamma-rays [12] acquired during the 1997 outburst of Mrk 501.

\section{Telescope hardware and data set}

The HEGRA IACT system consists of five telescopes, each with a tessellated mirror of $8.5 \mathrm{~m}^{2}$ area and $5 \mathrm{~m}$ focal length, and a 271-pixel camera with a $4.3^{\circ}$ field of view. The trigger condition requires a coincidence of two neighboring pixels above a threshold $q_{o}$ to trigger the camera, and a coincidence of triggers from two cameras to record the data. Details of the trigger system are given in [10]. Events are reconstructed by parameterizing the images using the Hillas image parameters, and by geometrically determining the direction and the impact point of the shower. A simple and relatively efficient method for cosmic-ray rejection is based on the difference in the width of $\gamma$-ray and cosmic-ray images, respectively. Width values are normalized to the mean width expected for a gamma-ray image of a given intensity and impact distance, and a mean scaled width is calculated by averaging over telescopes. The $\gamma$-ray showers exhibit, by definition, a mean scaled width around 1, whereas the broader cosmic-ray showers show larger values. A cut requiring a mean scaled width below 1.2 or 1.3 keeps virtually all gamma-rays and rejects a significant fraction of cosmic rays; a cut at 1.0 to 1.1 has lower gamma-ray acceptance, but optimizes the significance for the detection of $\gamma$-ray sources. The data analysis and performance of the system are described in more detail in $[11,12]$.

During 1997, when the data for this study were taken, only four of the five telescopes were included in the HEGRA IACT system; the fifth telescope (one of the corner telescopes) was still equipped with an older camera and was operated in stand-alone mode. The four telescopes can be used to emulate six different two-telescope stereo systems: three combinations with intertelescope distances around $70 \mathrm{~m}$ (from the central telescope to the three corner telescopes), two combinations with about $90 \mathrm{~m}$ and $110 \mathrm{~m}$ (the sides of the imperfect square), and one combination with $140 \mathrm{~m}$ (the diagonal). Data from pairs of (triggered) telescopes are analyzed, ignoring the information provided by the other telescopes. Since only two telescopes are required to trigger the system, there is no trigger bias or other influence from those other telescopes.

A slight difficulty arises since one compares combinations of different telescopes, rather than varying the distance between two given telescopes. While the four HEGRA system telescopes used here are identical in their construction, they differ somewhat in their age and hence the degree of mirror deterioration, in the quality of the alignment of the mirror tiles, and in the properties of the PMTs in the cameras, which show systematic variations between production 
batches. Different mirror reflectivities and PMT quantum efficiencies result in slightly different energy thresholds for given (identical) settings of electronics thresholds. The determination of image widths and hence the background rejection are sensitive to the quality of the mirror adjustment. These effects have to be determined from the data, and compensated.

The analysis is based on the data set recorded in 1997 during the outburst of Mrk 501. Only data during the high-flux periods between MJD 50595 and MJD 50613 were used. The data set was further restricted to small zenith angles, less than $20^{\circ}$, to approximate the situation for vertical showers. Mrk 501 was observed in the so-called wobble mode, with the source images $0.5^{\circ}$ away from the center of the cameras. An equivalent region imaged on the opposite side of the camera center was used as off-source region. Given the angular resolution of about $0.1^{\circ}$, the on-source and off-source regions are well separated.

\section{Analysis and results}

As a first step in the analysis, the detection rates were studied as a function of intertelescope distance.

To equalize the energy thresholds of all telescopes, pairs of telescopes were used to reconstruct showers and events with cores located at equal distance from both telescopes were selected. By comparing the mean size of the images in the two telescopes, and in particular by comparing the mean signal amplitude in the second-highest pixel (which determines if a telescopes triggers or not), and can derive correction factors which can be used to equalize the response of the telescopes. Three of the four telescopes were found to be identical within a few $\%$, one had a sensitivity which was lower by about 25\%. In the analysis, pixel amplitudes are corrected correspondingly, and only camera images with two pixels above 20 photoelectrons are accepted. This software threshold is high enough to eliminate any influence of the hardware threshold (at about 8 to 10 photoelectrons, depending on the data set), even after the worst-case $25 \%$ correction is applied.

The resulting detection rates of cosmic rays and of gamma-rays - after subtraction of the cosmicray background - are shown in Fig. 1. The rates measured for the three different telescope combinations around $70 \mathrm{~m}$ spacing agree within 5\%, indicating the precision in the adjustment of telescope thresholds. Detection rates decrease with increasing distance; between $70 \mathrm{~m}$ and $140 \mathrm{~m}$, rates drop by about $1 / 3$, with a very similar dependence for gamma rays as compared to cosmic rays. At first glance, this seems surprising, since compared to gamma-ray showers, the distribution of Cherenkov light in proton-induced showers - and hence the probability to trigger - is more strongly peaked near the shower axis, favoring a smaller separation of telescopes. On the other hand, however, the trigger probability for gamma-ray showers near threshold - where most of the detection rate originates - cuts off sharply around $120 \mathrm{~m}$ to $130 \mathrm{~m}$, whereas proton-induced showers occasionally trigger at larger distances [13]. The two effects compensate each other to a certain extent. In addition, about $33 \%$ of the cosmic-ray triggers are caused by primaries heavier than protons [14]. These particles interact higher in 


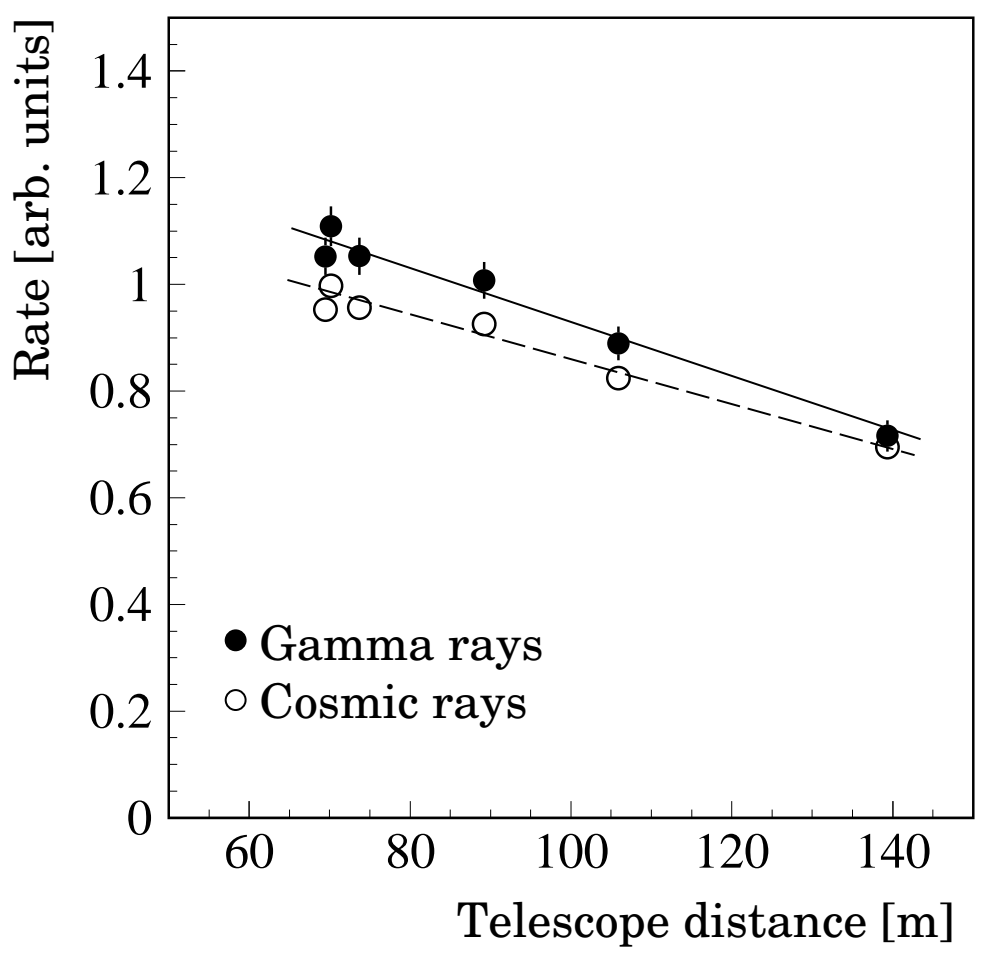

Fig. 1. Detection rates of cosmic rays and of gamma rays as a function of the spacing between stereoscopic pairs of telescopes, using a software trigger requirement of more than 20 photoelectrons in two pixels of both cameras. The normalization is arbitrary. Gamma-ray events were selected using very loose cuts on pointing relative to Mrk $501\left(<0.45^{\circ}\right)$ and on the mean scaled width of the images $(<1.3)$, keeping essentially all gamma-rays. Cosmic-ray background in the on-source sample was subtracted on a statistical basis. The lines are drawn to guide the eye.

the atmosphere and produce a wider light pool, again enhancing the rate for telescopes with wide spacing. In the later analysis, shape cuts completely eliminate such events. Indeed, if only cosmic-ray events with narrow (proton- or gamma-like) images are accepted, their rates fall off steeper with distance than the all-inclusive rate, or the gamma-ray rate.

As a measure of the sensitivity for weak sources, the ratio $S / \sqrt{B}$ of the gamma-ray rate to the square root of the cosmic-ray rate was used. The significance of background-dominated signals scales with this ratio. For optimum sensitivity, $S / \sqrt{B}$ is optimized by tighter cuts on the pointing of showers, and on the image shapes. Fig. 2 shows the angular resolution provided by pairs of telescopes as a function of spacing. The three pairs at $70 \mathrm{~m}$ show differences at the level of $10 \%$, indicating small differences in the quality of the mirror alignment and of the telescope alignment. Angular resolution improves slightly with increasing spacing; however, given the $10 \%$ systematic variations, this effect is of marginal significance.

To determine the sensitivity for the different combinations of telescopes, cuts on pointing and on the mean scaled width were optimized for each combination, resulting in pointing cuts around $0.1^{\circ}$ and cuts on the mean scaled width around 1.05. In addition, a lower limit of 0.75 was imposed on the mean scaled width. Due to differences in the quality of the mirror alignment, the enhancement of significance due to such cuts differs slightly between telescopes. This can be 


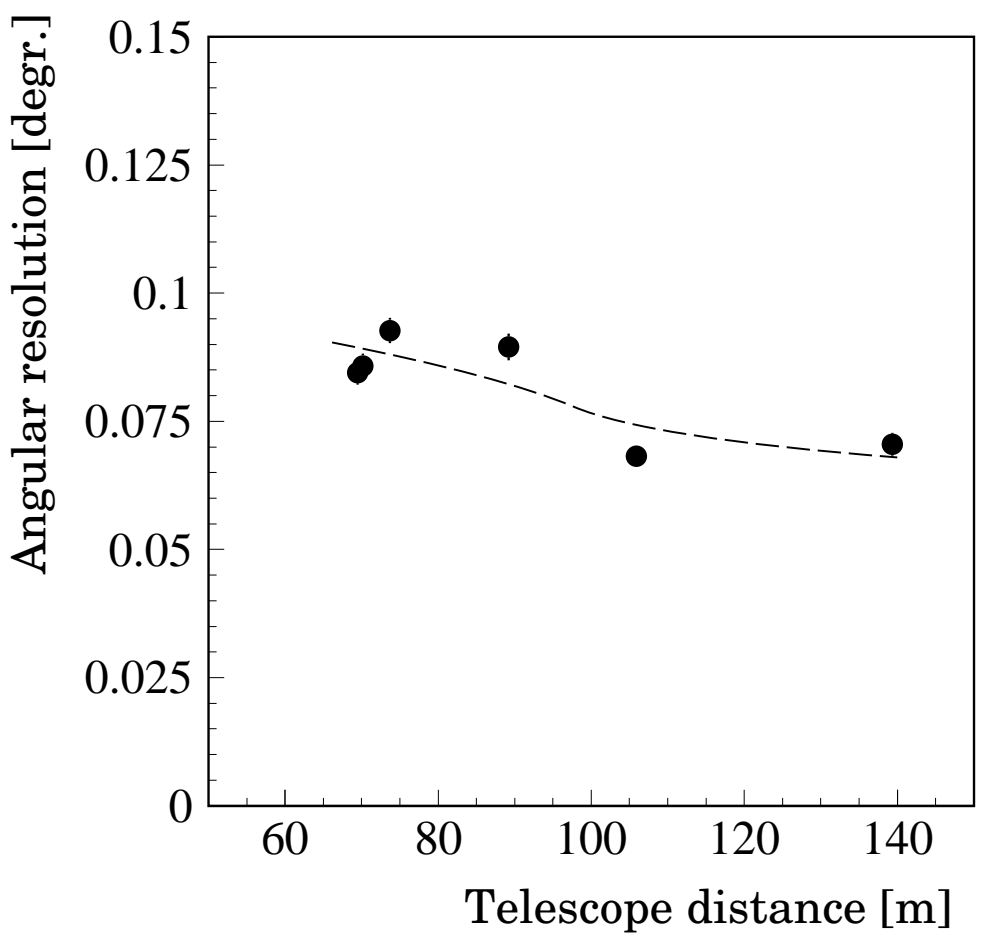

Fig. 2. Angular resolution provided by pairs of telescopes as a function of telescope spacing. A software trigger threshold of 20 photoelectrons is used. The angular resolution is determined by fitting a Gaussian to the difference between shower direction and direction to the source, projected on two orthogonal axes. The dashed line is drawn to guide the eye.

studied by selecting events with telescopes $A$ and $B$, and deriving the significance of the signal by cutting only on the event shape in $A$, or only in $B$. For identical telescopes, the results should be identical. Based on such studies, sensitivity corrections for the different telescope pairs were derived; these correction factors were always below 10\%. Fig. 3(a) shows the resulting significance (defined as $S / \sqrt{B}$ ) of the Mrk 501 signal. The errors shown are statistical, and are dominated by the low statistics in the cosmic-ray background sample after cuts (even though the background region was chosen to be larger than the signal region). One finds that the significance is almost independent of telescope spacing, over the $70 \mathrm{~m}$ to $140 \mathrm{~m}$ range covered.

Concerning systematic uncertainties, we note that the results for the three combinations at $70 \mathrm{~m}$ agree reasonably well. Variations of parameters such as the software trigger threshold, or the exact values of the pointing cuts and angular cuts produce stable results, with systematic variations of at most $10 \%$.

The absolute significance of a point source detected with two stereoscopic IACTs, and its dependence on the intertelescope distance will, of course, depend on additional cuts which may be applied in the analysis. Additional requirements may, for example, concern the stereo angle, defined as the angle between the two views of the shower axis provided by the two telescopes, or, equivalently, the angle between the image axes in the two cameras. If the stereo angle is small - as it is the case for events with shower impact points on or near the line connecting the two telescopes, and for very distant showers - the two views coincide and the 

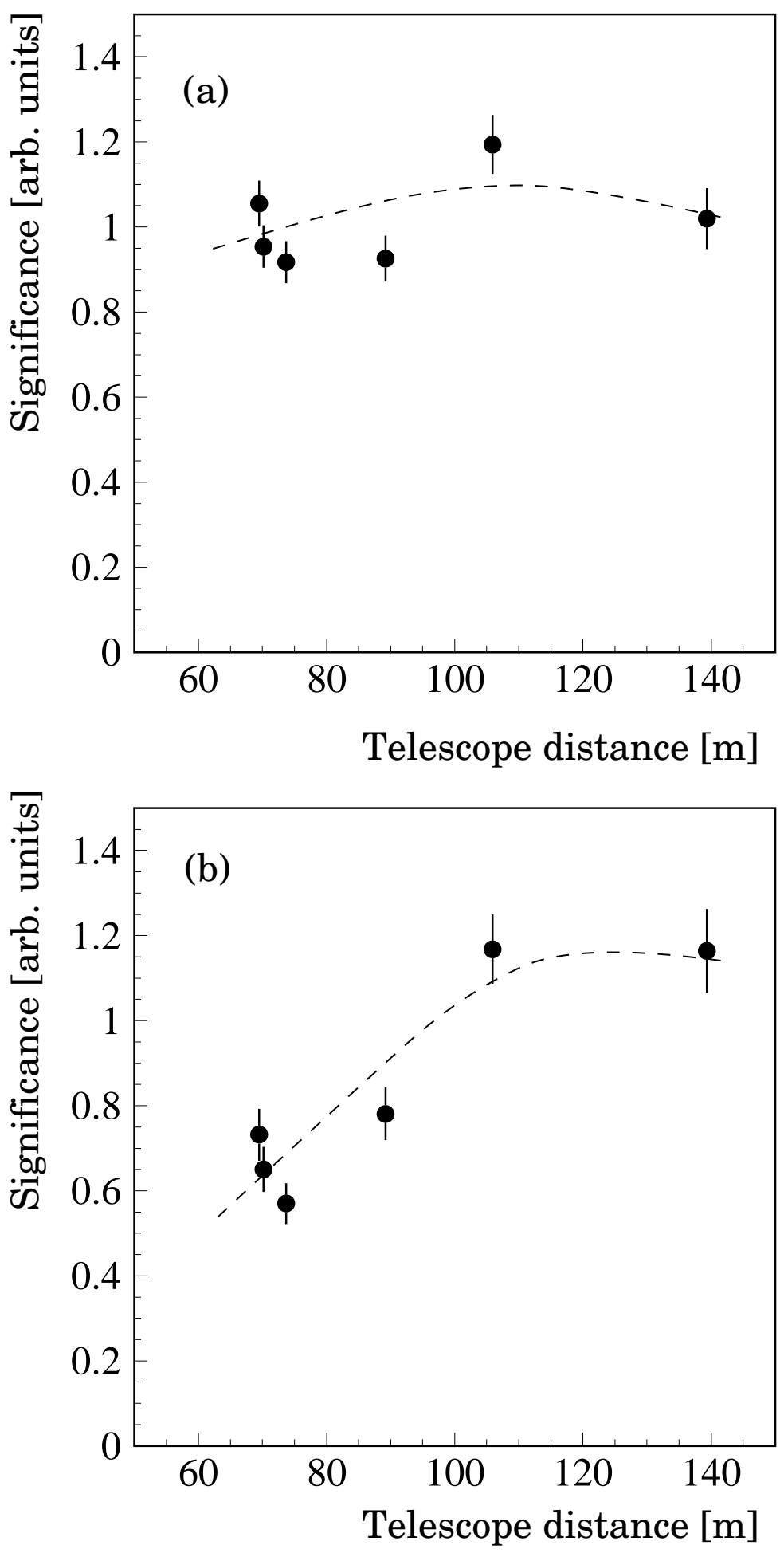

Fig. 3. (a) Significance $S / \sqrt{B}$ of the Mrk 501 signal for different pairs of telescopes, with optimized cuts on shower pointing and image shapes in the two telescopes. The significance is normalized to $\approx 1$ for the $70 \mathrm{~m}$ spacing. The dashed line is drawn to guide the eye. (b) As (a), but with an additional cut of a stereo angle of at least $45^{\circ}$ between the two views. The normalization if the same as for (a), so that the two data sets can be compared directly. A weaker $20^{\circ}$ cut on the stereo angle, as used in some HEGRA analyses, has little effect on the significance, and yields the almost same results as shown in (a). 
spatial reconstruction of the shower axis is difficult. In some analyses, one will therefore add the requirement of a minimal stereo angle between the two views, in order to increase the reliability of the stereoscopic reconstruction. For a minimum stereo angle of $20^{\circ}-$ as used in some HEGRA work - the resulting significance remains virtually unchanged compared to the data shown in Fig. 3(a). With a large minimum stereo angle of $45^{\circ}$ (Fig. 3(b)), the distance dependence becomes more pronounced; for small telescope distances, significance is reduced, while for large distances it remains or is even slightly enhanced. The explanation is simple. For a telescope spacing $d$ and a stereo angle greater than $45^{\circ}$, shower cores are accepted at most up to a distance of $\approx 1.2 d$ from the center of the telescope pair. As long as this distance is below the radius of the light pool, the effective detection area is reduced. One concludes that such a cut might be beneficial to reduce systematic effects in spectral measurements, for example, but should not be applied in searches for gamma-ray sources.

Another quantity which influences the distance dependence is the field of view of the cameras. Cameras with small field of view will perform worse for large distances. For example, rejecting events with image centroids beyond $1^{\circ}$ from the camera center reduces the sensitivity for the $140 \mathrm{~m}$ point by a factor of about 2 .

\section{Summary}

The performance of two-telescope stereoscopic IACT systems was studied experimentally using the HEGRA telescopes, as a function of telescope spacing in the range between $70 \mathrm{~m}$ and $140 \mathrm{~m}$. While detection rates decrease with increasing spacing, the significance of source signals is almost independent of distance, with a slight improvement for distances beyond $100 \mathrm{~m}$. These results confirm Monte Carlo simulations (see, for example, [4]) which generally show that the exact choice of telescope spacing is not a very critical parameter in the design of IACT systems (provided that the field of view of the cameras is sufficiently large not to limit the range of shower impact parameters). An analysis requirement of a minimum angle between views favors larger distances.

The results shown apply, strictly speaking, only to two-telescope systems. In IACT arrays with a large number of telescopes, one may place telescopes at maximum spacing in order to maximize the effective area of the array, under the condition that most individual showers are observed by two or maybe three or four telescopes. Alternatively one may choose to place telescopes close to each other, such that an individual shower is observed simultaneously by almost all telescopes, improving the quality of the reconstruction and lowering the threshold, at the expense of detection area at energies well above threshold. In the first case, one would probably use a spacing between adjacent telescopes between $100 \mathrm{~m}$ and $150 \mathrm{~m}$; in the second case, one would limit the maximum spacing between any pair of telescopes to this distance. 


\section{Acknowledgements}

The support of the HEGRA experiment by the German Ministry for Research and Technology BMBF and by the Spanish Research Council CYCIT is acknowledged. We are grateful to the Instituto de Astrofisica de Canarias for the use of the site and for providing excellent working conditions. We gratefully acknowledge the technical support staff of Heidelberg, Kiel, Munich, and Yerevan.

\section{References}

[1] VERITAS Letter of Intent, T.C. Weekes et al. (1997); VERITAS Proposal (1999).

[2] HESS Letter of Intent, F. Aharonian et al. (1997).

[3] F.A. Aharonian et al., Astroparticle Phys. 6 (1997) 343.

[4] A. Konopelko, Astropart. Phys., in press, and astro-ph/9901365.

[5] R. Lamb, Proceedings of the Int. Workshop "Towards a Major Atmospheric Cherenkov Detector IV”, Padua, (1995), M. Cresti (Ed.), p. 386; F. Krennrich et al., Astropart. Phys. 8 (1998) 213.

[6] N. Hayashida et al., Astroph. J. 504 (1998) L71; T. Yamamoto et al., Astropart. Phys. 11 (1999) 141.

[7] A. Konopelko, Proceedings of the Int. Workshop "Towards a Major Atmospheric Cherenkov Detector V", Kruger Park, (1997),p. 208.

[8] A. Konopelko et al., Astropart.Phys. 10 (1999) 275.

[9] F. Aharonian et al., Astropart.Phys. 10 (1999) 21.

[10] N. Bulian et al., Astroparticle Phys. 8 1998) 223.

[11] A. Daum et al., Astroparticle Phys. 8 (1997) 1.

[12] F.A. Aharonian et al., Astron. Astrophys. 342 (1999) 69.

[13] C. Köhler et al., Astropart. Phys. 6 (1996) 77.

[14] A.V. Plyasheshnikov et al., J. Phys. G 24 (1998) 653. 